\title{
Technological processes intensification in devices with magneto-fluidized bed
}

\author{
Ivan Egorov ${ }^{1, *}$, Nikolay Egorov ${ }^{2}$ \\ ${ }^{1}$ Don State Technical University, Rostov-on-Don, Russian Federation \\ ${ }^{2}$ Southern Federal University, Rostov-on-Don, Russian Federation
}

\begin{abstract}
The paper shows the efficiency of application of magneto-fluidized bed formed of ferromagnetic materials in press-forms dosed filling and milling devices. For powders without natural fluidity, dosing device is situated between electromagnets creating constant magnetic field with horizontal induction lines and alternating gradient magnetic field with vertical induction lines and higher induction gradient in the lowest part of chamber with powder. This configuration allows development of uniform efflux speed of ferromagnetic powders from $2 \mathrm{~mm}$ diameter opening. For example, mass efflux speed of strontium ferrite powder with $1 \mu \mathrm{m}$ average particle size in magneto-fluidized bed reached $181.1 \mathrm{mg} / \mathrm{s}$ and the dosing time of $2000 \mathrm{mg}$ of this powder was $11.1 \mathrm{~s}$. For practical usage of magneto-fluidized bed in the milling device, beater mill is situated between electromagnet poles in the way that induction lines of constant and alternating gradient magnetic fields were mutually perpendicular and parallel to the plane of rotating beaters. Milling of particulate strontium ferrite with $1558.5 \mu \mathrm{m}$ average particle size during 120 minutes in magneto-fluidized bed allows increasing milling degree in 16.2 times by comparison with processing without electromagnetic effect.
\end{abstract}

\section{Introduction}

Powder metallurgy uses various particulate metals and alloys as raw materials. Particle size decrease increases interparticle interaction leading to changing of particulate system technological characteristics [1]. Considerable theoretical and experimental material about influence of magnetic fields on rheological state of magnetic fluids [2-4] and particulate materials in fluidized state [5-7] was accumulated. There is a wide range of devices that fluidize particulate medium by means of hydration, aeration, ultrasonic, electrodynamic and electromagnetic effects [8-11]. In case of ferromagnetic materials electromagnetic effect on particulate medium (uniform alternating magnetic field, rotating magnetic field, superposition of alternating gradient and constant magnetic fields [12-15]) is the most effective way of obtaining dynamically stable weighted state of particulate system - magneto fluidized bed. Using of magneto-fluidized bed can largely intensify technological processes of mixing, milling, dosing, drying etc. [16-19].

For practical use of magneto-fluidized bed, ferromagnetic powder is placed in working chamber situated between poles of electromagnets forming constant and alternating gradient magnetic fields. Disposition of working chamber, poles of electromagnets and their configuration depend on required character of particle motion in the process.

\section{Problem}

Ferromagnetic powders don't have natural fluidity (don't flow through an opening in bunker to press-form) as their particles form aggregates and they stuck in the opening. Magneto-fluidized bed allows to destroy particles aggregates and greatly reduce interparticular friction. This makes possible flowing of powder through bunker opening.

In case of dry milling particle aggregates are also a problem as large part of energy goes to aggregate destruction and small particles that surround large ones protect them from milling effect. This problem can be partially solved by powder fluidization as it destructs particle aggregates.

The goal of the work is in development of determination principles of the most effective regimes and required configuration of alternating gradient and constant magnetic fields for intensification of technological processes of dozed press-form filling and milling in rheological controlled fluidized bed.

\section{Experiment}

In alternating gradient and constant magnetic fields depending on regimes of electromagnetic effect on ferromagnetic powder, take place processes of aggregate destruction as well as formation of secondary aggregates.

\footnotetext{
* Corresponding author: egorovivan1@gmail.com
} 
Direction of particle motion in magneto-fluidized bed depends on electromagnets poles configuration, values of constant field induction and alternating field gradient [20] and is conditioned by requirements to the device. Fig. 1 presents diagram of device for development of ferromagnetic powders stimulated fluidity. Poles of direct current electromagnet 1 form magnetic field with horizontal induction lines. Poles of alternating current electromagnet 2 form magnetic field with vertical induction lines with higher induction gradient below working chamber 3. For development of high gradient, we sharpened one of alternating current electromagnet poles. Working chamber contains wire mesh 4 to prevent clogging of efflux opening with large particles. To develop stimulated fluidity through efflux opening 5 with diameter $2 \mathrm{~mm}$ in the receiving chamber 6 , the magneto fluidized bed is formed by the effect of magnetic fields. In order to minimize arched effects, we used working chamber with flat bottom. Higher gradient of alternating magnetic field below efflux opening makes ferromagnetic particles flow to the receiving chamber.

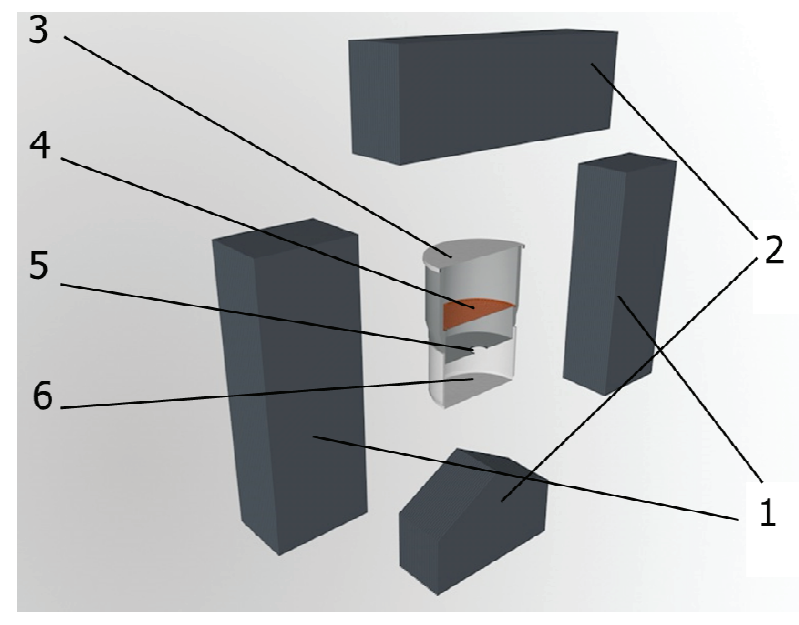

Fig. 1. Diagram of device for ferromagnetic powders stimulated fluidity development: 1 - direct current electromagnet poles, 2 - alternating current electromagnet poles, 3 - working chamber, 4 - wire mesh, 5 - efflux opening, 6 - receiving chamber

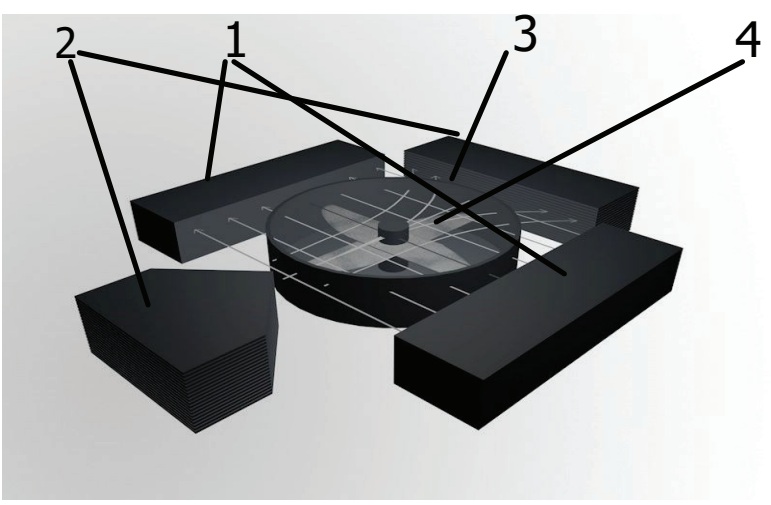

Fig. 2. Diagram of ferromagnetic material milling device: 1 - direct current electromagnet poles, 2 - alternating current electromagnet poles, 3 - beater mill, 4 - beaters
Fig. 2 presents diagram of device for milling particulate ferromagnetic materials. Between the poles of direct current electromagnet 1 and alternating current is located beater mill 3 with nonmagnetic body. Induction lines of constant and alternating gradient magnetic fields are mutually perpendicular and parallel to the plane of rotating with $15000 \mathrm{rpm}$ beaters 4. Dynamics of magneto-fluidized bed of particles and aggregates in beater mill determinates the character of impact interactions of these elements with rotating beaters and between themselves. Large particles are destructed by rotating beaters, smaller ones have less probability to be hit by beaters but are restlessly hit by other particles, that leads to self-milling. An important condition for development of magneto-fluidized bed during milling of particulate ferromagnetic material is the intense particle motion providing intensification of the technological process.

At given configuration of magnetic fields for making choice of the most effective electromagnetic effect regimes in each case of practical realization one need to pick up the ratio of constant magnetic field induction and alternating field induction gradient providing rheological features of magneto-fluidized bed.

\section{Results and discussion}

Significant demands at continuous processes of dosing, press-form filling and fabrication of permanent magnets are made to provision of constant efflux speed of particulate material from opening, because dosing error influences quality of final product [21].

Fig. 3 presents experimental dependencies of mass efflux speed of barium ferrite powder with $1 \mu \mathrm{m}$ average particle size (a) and of strontium ferrite with $1 \mu \mathrm{m}$ (b) and $50 \mu \mathrm{m}(\mathrm{c})$ average particle size through opening with $2 \mathrm{~mm}$ diameter from constant magnetic field induction at fixed values of alternating magnetic field induction gradient. Presented dependencies show that ferrite powders mass efflux speed grows with increase of induction gradient at fixed constant magnetic field induction. This can be explained by considering that in gradient alternating magnetic field the force proportional to the induction gradient acts on particles of ferromagnetic material. In case of barium ferrite powder with $1 \mu \mathrm{m}$ average particle size (Fig. 3a) in the field with induction gradient $250 \mathrm{mT} / \mathrm{m}$ mass efflux speed increases to $34.3 \mathrm{mg} / \mathrm{s}$ when increasing constant magnetic field induction $\left(\mathrm{B}_{\mathrm{c}}\right)$ to $6.8 \mathrm{mT}$. Further $\mathrm{B}_{\mathrm{c}}$ increase leads to mass efflux speed decrease as high constant magnetic field begin to hold particles in the area of its induction lines. Induction gradient increase to 593 $\mathrm{mT} / \mathrm{m}$ at $B_{\mathrm{c}}=15.7 \mathrm{mT}$ makes mass efflux speed increase to $96.6 \mathrm{mg} / \mathrm{s}$. In case of strontium ferrite with $1 \mu \mathrm{m}$ average particle size in field with induction gradients of 250 and $593 \mathrm{mT} / \mathrm{m}$ (Fig. 3b) maximal values of efflux speed are 97.4 and $181.1 \mathrm{mg} / \mathrm{s}$, correspondingly. For the same induction gradient values in case of strontium ferrite $50 \mu \mathrm{m}$ average particle size (Fig. 3c) values of corresponding speeds are 83.4 and $143.4 \mathrm{mg} / \mathrm{s}$. 

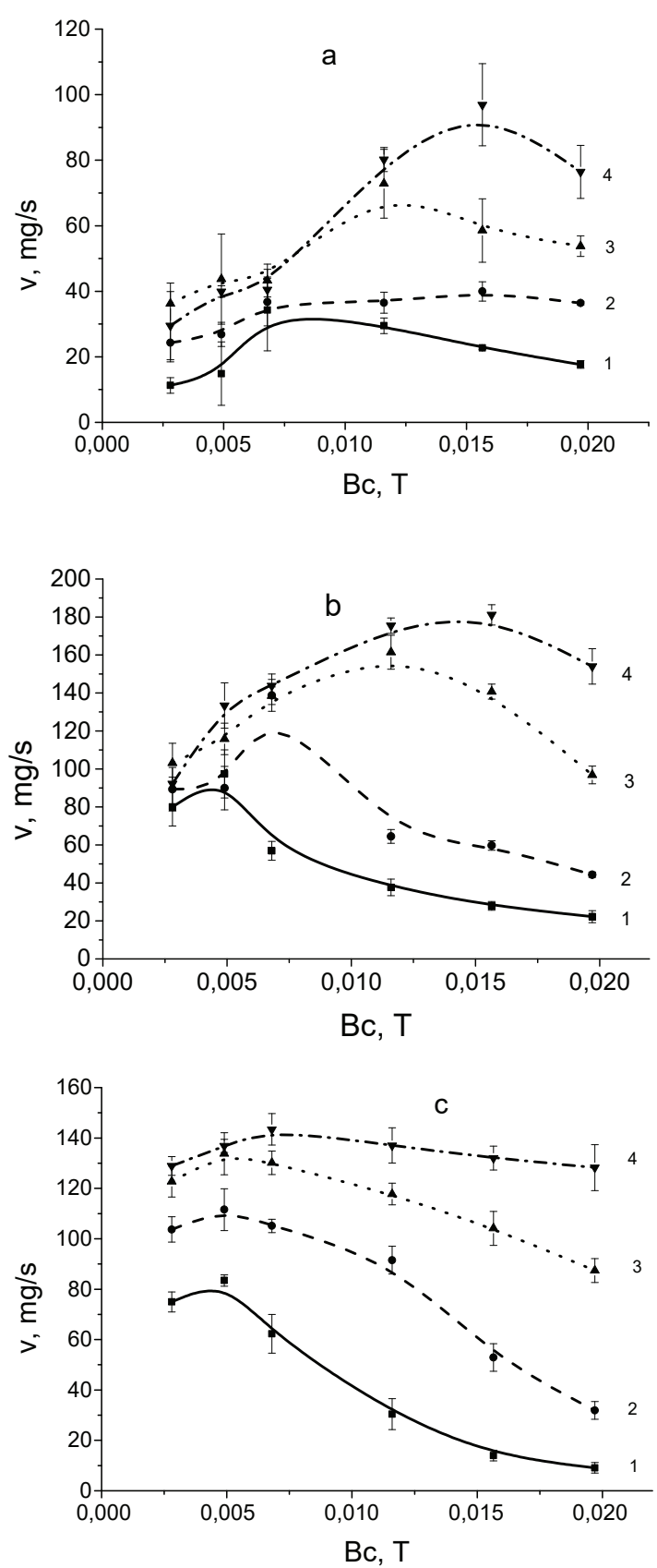

Fig. 3. Dependencies of mass efflux speed of barium ferrite (a) and strontium ferrite $(b, c)$ powders through $2 \mathrm{~mm}$ diameter opening from constant magnetic field induction at various alternating field induction gradient values: $1-250 \mathrm{mT} / \mathrm{m}$, 2 - $390 \mathrm{mT} / \mathrm{m}, 3$ - $525 \mathrm{mT} / \mathrm{m}, 4$ - $593 \mathrm{mT} / \mathrm{m}$

Obtained results show that to fabricate permanent magnet with mass of $2000 \mathrm{mg}$ from barium ferrite and strontium ferrite powders with $1 \mu \mathrm{m}$ average particle size in regime of $\mathrm{B}_{\mathrm{c}}=15.7 \mathrm{mT}$ and induction gradient 390 $\mathrm{mT} / \mathrm{m}$ dosing time will be 50.0 and 33.3 seconds correspondingly. In case of induction gradient increase to $593 \mathrm{mT} / \mathrm{m}$, dosing time will decrease to 20.0 and 11.1 seconds correspondingly.

Main drawbacks at powder charge preparation for pressing in existing ferrite production are: long milling duration, large energy consumption and poor reproducibility of particle size distribution that leads to variance of permanent magnet parameters [22].

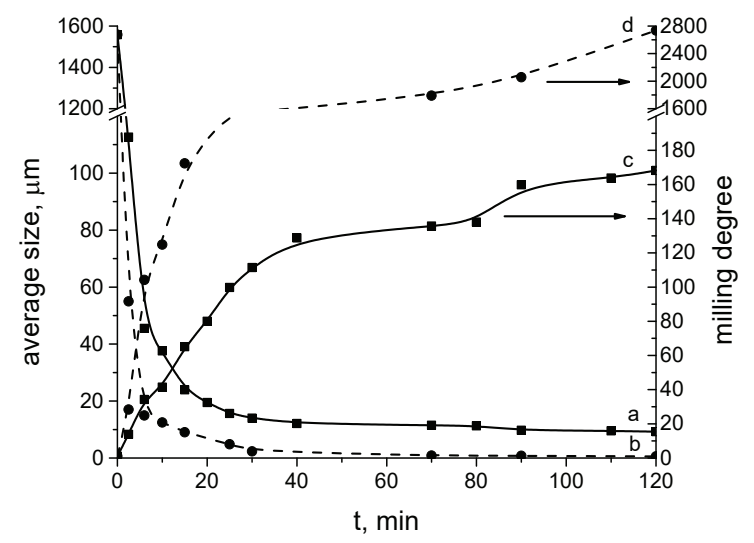

Fig. 4. Dependence of average particle size and milling degree of strontium ferrite powder from milling time without electromagnetic effect $(a, c)$ and in magneto fluidized bed $(b, d)$

Fig. 4 presents experimental dependencies of average particle size and milling degree (ration of initial average particle size to current particle size) from milling time in beater mill without electromagnetic effect and in magneto fluidized bed, formed in constant magnetic field with induction of $15.3 \mathrm{mT}$ and in alternating one with induction gradient of $90 \mathrm{mT} / \mathrm{m}$. Particulate strontium ferrite with average particle size $1558.5 \mu \mathrm{m}$, maximal size of $3100 \mu \mathrm{m}$, median of $1477 \mu \mathrm{m}$ was milled. In case of processing initial particulate material in mill during 120 minutes without electromagnetic effect, average particle size decreased to $9.26 \mu \mathrm{m}$ and in magnetofluidized bed to $0.57 \mu \mathrm{m}$, milling degree increased in 16.2 times, that witness of milling intensification in magneto-fluidized bed.

The increase of milling efficiency is archived through holding particulate medium in rotating beaters area and self-milling of particles as a result of collisions of particles with each other in alternating gradient magnetic field.

On early milling stages beaters make almost all job, but the less particle size becomes the less effective they become. Rotating beaters don't mix powder in the chmber enough, some of it lies on the bottom slightly involved in the milling process. Magneto-fluidized bed develop additional mizing that involves all the powder into milling process.

Calculations show that value of particle kinetic energy obtained in magneto-fluidized bed is not enough for particle destruction in the interparticle collision act. However, despite insufficient speeds of particle motion in magneto-fluidized bed, intensive self-milling takes place there. Multiple interparticle collisions cause fatigue destruction of powder particles. Processing of strontium ferrite particulate material with $81.3 \mu \mathrm{m}$ average particle size only in magneto-fluidized bed formed in constant magnetic field with induction of 15.4 $\mathrm{mT}$ and alternating magnetic field with frequency $50 \mathrm{~Hz}$ and induction gradient of $90.0 \mathrm{mT} / \mathrm{m}$, allowed to decrease dispersed composition of obtained product. Quality of particles smaller than $5.5 \mu \mathrm{m}$ contained in initial powder increased in 31 times [23] after 70 minutes of processing. In this way, intensification of 
milling process in magneto fluidized bed is conditioned by self-milling and mixing of particulate material.

Milling in magneto-fluidized bed require additional energy consumption on power supply of electromagnets. However, getting strontium ferrite powder with $9 \mu \mathrm{m}$ average particle size from initial $1558.5 \mu \mathrm{m}$ requires 130 minutes and the $15000 \mathrm{~J}$ of energy. In case of milling in magneto fluidized bed for getting the same average particle size requires 15 minutes and the energy constitutes only $3126 \mathrm{~J}$.

Presented results show that application of magnetofluidized bed to milling of particulate ferromagnetic materials not only intensifies the process but also decreases its energy consumption.

\section{Conclusion}

The paper experimentally substantiates effectiveness of application of magneto-fluidized bed of particulate ferrite materials in devices of dosed press-form filling and milling.

Application of magneto-fluidized bed to press-forms filling process allows developing steady efflux of ferromagnetic powders without natural fluidity. Electromagnets configuration required for the process is horizontal constant magnetic field and vertical alternating magnetic field with high gradient below efflux opening. High and steady efflux speed is archieved at constant magnetic field induction about $15 \mathrm{mT}$ (for $1 \mu \mathrm{m}$ barium and strontium ferrite powders) and alternating field induction gradient about $600 \mathrm{mT} / \mathrm{m}$. This allow to develop automated dosing devices for press-forms filling and futher pressing of powder charge into permanent magnet blank.

Application of magneto-fluidized bed to the process of particulate ferrite milling in beater mill allows dry milling process to average powder particle size less than a micrometer. This is unattainable at the same mill without electromagnetic effect. Development of magneto-fluidized bed holds powder particles around rotating beaters making all particles involved in the milling process. Restless interparticle collisions, though small, but develop fatigue stresses that cause particle fatigue destruction (like in vibrating mill).

Analysis of obtained results shows that the choice of magnetic fields configuration optimal for specific process and determination of most rational regimes to develop predetermined rheological state of particulate system in magneto-fluidized bed allow to greatly intensify technological processes.

The work was supported by grant of RFFI № 16-38-00365.

\section{References}

1. J.M. Valverde, Springer Netherlands, 136 (2013)

2. J. De Vicente, D.J. Klingenberg, R. HidalgoAlvarez, Soft Matter 73701 (2001)

3. H. See, Rheologica Acta 42, 86 (2003)
4. T. Gadipelly, S. Rajender, Physica B: Condensed Matter 448, 346 (2014)

5. J.Y. Hristov, 5th European Rheology Conference, (Portoroz, Slovenia, 1998)

6. M.J. Espin, M.A.S. Quintanilla, J.M. Valverde, Chem. Eng. J. 277, 269 (2015)

7. M.J. Espin, J.M. Valverde, M.A.S. Quintanilla, J. of Fluid Mech. 732, 282 (2013)

8. I.B. Uriev, N.V. Mikhaylov, P.A. Rebinder, Rep. of AS of USSR 184, 192 (1969) [in Russian]

9. I.M. Kirko, M.V. Filippov, J. Tech. Phys. 30, 1081 (1960) [in Russian]

10. P.A. Kouzov, Basics of analysis of disperse compound of industrial dusts and milled materials, 264 (1987) [in Russian]

11. O.A. Myazdrikov, Electrodynamic fluidizing of particulate systems, 160 (1984) [in Russian]

12. A.P. Lupanov, A.Yu. Vinarov, A.P. Kanavin, V.I. Panteleev, J. Tech. Phys. 77, 134 (2007) [in Russian]

13. Yu. A. Buevich, S.V. Syutkin, V.V. Tetyuhin, Magn. Hydrodyn. 4, 3 (1984) [in Russian]

14. M.K. Bologa, I.F. Marta, Magn. Hydrodyn. 3, 103 (1988) [in Russian]

15. I.N. Egorov, S.I. Egorova, Russ. J. Non_Ferr. Met. 55, 371 (2014) [in Russian]

16. I.N. Egorov, S.I. Egorova, A.I. Chernyi, Pat 022640 (EA, 2016)

17. I.N. Egorov, S.I. Egorova, Yu.M. Vernigorov, Pat 2306180 (RF, 2007)

18. T. Chou, G. Nishizawa, M. Hatakeyama, C. Ishizaka, Pat 7416613 (US, 2008)

19. S. Okumura, A. Nakamura, Pat. 2003/0209842 (US, 2003)

20. I.N. Egorov, S.I. Egorova, G.F. Lemeshko, Perspective directions of developing finishing methods of workpiece processing (Symp. Eng. Techn., Rostov-on-Don, 2016) [in Russian].

21. A.M. Stryzhankov, P.F. Pool, Pat 1253738 (USSR, 1986).

22. D.D. Logvinenko, O.P. Shelyakov, Intensification of technological processes in devices with vortex layer (1976) [in Russian].

23. N.Ya. Egorov, I.N. Egorov, V.P. Kryzhanovsky, A.I. Chernyi, Int. sc. Inst. "EDUCATIO", 2, 41 (2015) [in Russian]. 